\title{
Anti-allodynic effect of interleukin 10 in a mouse model of complex regional pain syndrome through reduction of NKI receptor expression of microglia in the spinal cord
}

This article was published in the following Dove Press journal: Journal of Pain Research

Jong-Heon Kim'

Jin-Sung Park ${ }^{2}$

Donghwi Park ${ }^{3,4}$

'Department of Pharmacology, Brain Science and Engineering Institute, School of Medicine, Kyungpook National University, Daegu, Republic of Korea;

${ }^{2}$ Department of Neurology, School of Medicine, Kyungpook National University Chilgok Hospital, Daegu, Republic of Korea; ${ }^{3}$ Department of Rehabilitation Medicine, Daegu Fatima Hospital, Daegu, Republic of Korea; ${ }^{4}$ Department of Pharmacology, School of Medicine, Kyungpook National University, Daegu, Republic of Korea
Correspondence: Donghwi Park Department of Rehabilitation Medicine, Daegu Fatima Hospital, Ayangro 99,

Dong gu, Daegu 4I 199, Republic of Korea

Tel +82 539407821

Fax +82 539547417

Email bdome@hanmail.net
Background: To date, there has been no study on the effects of interleukin-10 (IL-10) on complex regional pain syndrome (CRPS) rodent models, despite the anti-allodynic effect of IL-10 in previous studies. Thus, the aim of this study was to investigate the effect of IL-10 in a CRPS mouse model and find whether early inhibition of neuro-inflammation by IL-10 administration, which is considered to be one of the important mechanisms in the generation of central sensitization, could prevent the transition from the acute stage to the chronic stage of CRPS.

Method: A mouse model of CRPS ( $n=6 /$ group) involving tibia fracture/cast immobilization to test the efficacy of intrathecal IL-10 $\left(0.3 \mu \mathrm{g} / 5 \mu \mathrm{L}^{-1}\right.$ day $^{-1}$ for 7 days $)$ or vehicle during the acute (3 weeks after fracture) stage of CRPS.

Results: Intrathecal recombinant IL-10 (rIL-10) administration was anti-allodynic in the acute stage of the CRPS mouse model, and these anti-allodynic effects of IL-10 developed by modulating microglial activation and decreasing NK1 receptor expression in the spinal cord. However, intrathecal rIL-10 administration in the acute stage of the CRPS mouse model cannot prevent the transition to the chronic stage of CRPS in the acute stage of CRPS.

Conclusion: Collectively, these results demonstrate that intrathecally administered rIL-10 attenuates mechanical allodynia in the CRPS mouse model. However, this effect of IL-10 on allodynia in the acute stage of CRPS was not sufficient to prevent the transition to the chronic stage of CRPS. In the future, further studies about the mechanisms of central sensitization in CRPS will be necessary.

Keywords: CRPS, IL-10, microglia, glial activation, NK1 receptor, substance P

\section{Introduction}

Complex regional pain syndrome (CRPS) is a painful, disabling, and often chronic condition with an estimated incidence rate of 26.2 per 100,000 person years. ${ }^{1}$ The syndrome encompasses a disparate collection of symptoms and signs involving hypersensitivity to noxious somatosensory stimuli (hyperalgesia), pain from non-noxious stimuli (allodynia), spreading pain (spontaneous and evoked) that involves undamaged regions in the affected limb to the opposite limb and other parts of the body, dysfunction of skin growth changes, autonomic nervous systems, bone demineralization, and vascular dysfunction as well as changes in emotional and cognitive functions. ${ }^{2}$

Although CRPS is described as a single disease, it is usually categorized into two distinct phases: an acute and a chronic stage of CRPS. ${ }^{3}$ In addition to changes in the clinical symptoms and signs, these two distinct stages are accompanied by different 
biochemical changes in both human patients ${ }^{4}$ and mouse models of CRPS. ${ }^{5}$ This transition from the acute to the chronic stage of CRPS has been investigated through previous studies, which have found that it may be related to a change in the underlying mechanisms of pain, with peripheral mechanisms believed to support at least some of the acute manifestations and changes of the central nervous system changing to support symptoms in the more chronic stages. Although the mechanisms supporting the chronic phases of CRPS are still very poorly understood, neuro-inflammation by glia cells has been identified as one of the major causes of central sensitization, and regarded as one of the causes of the chronic stage of CRPS in previous studies. ${ }^{6-8}$

Interleukin-10 (IL-10) is known to inhibit neuro-inflammation by modulating the activation of glia cells,,${ }^{9,10}$ and to improve neuropathic pain, such as allodynia in the rodent neuropathic pain model. ${ }^{11-13}$ Despite the therapeutic potency of IL-10, to the best of our knowledge, there has been no study about the effects of IL-10 on CRPS rodent models. In this study, therefore, we investigated the therapeutic effect of IL-10 on pain in a CRPS mouse model, as well as the central sensitization by neuro-inflammation, which is considered to be one of the main mechanisms of the transition to the chronic stage of CRPS from the acute stage.

\section{Method}

\section{Animals}

Male C57/B6J mice between 12-14 weeks of age were obtained from KOATECH Experimental Animal Center (Pyeongtaek, South Korea) and were allowed to habituate to the animal facility for at least 10 days prior to the experiments. The mice were housed in four groups on a 12-hour dark-light cycle, in an ambient temperature in the range of $20.0^{\circ} \mathrm{C}$ to $22.0^{\circ} \mathrm{C}$, and fed as desired. The animal experiments were approved by the Institutional Animal Care Committee of Daegu Fatima Hospital and conducted in accordance with the animal care guidelines of the National Institutes of Health. All efforts were made to minimize animal suffering and the number of animals used.

\section{Limb fracture and cast immobilization}

After random allocation to the fracture/cast group or the control group, mice were anesthetized with $1.5 \%$ isoflurane and a tibial fracture of the left limb was performed. We made a closed fracture of the left tibia just distal to the middle of the tibia using a hemostat, and the left leg was wrapped in casting tapes, as previously described. ${ }^{2}$ After the procedure, subcutaneous enrofloxacin $(5 \mathrm{mg} / \mathrm{kg})$, tramadol (30 mg/kg), and normal saline (1.5 mL once) were injected for the next 2 days, for management of postoperative pain, prevention of dehydration, and infection. After 3 weeks of surgery, the casts were removed under isoflurane anesthesia. Only mice that showed typical symptoms of CRPS, hind paw swelling, and allodynia, were selected as CRPS model. A total of 50 mice were operated on, and 13 of them without symptoms of CRPS were excluded. Naïve sex- and age-matched mice were used as controls.

\section{Drug preparations}

Control and fracture mice were randomly assigned to one of two treatment groups: IL-10 or vehicle infusion. Mouse IL-10 (I3019) was purchased from Sigma, (St Louis, MO, USA). Recombinant mouse IL-10 was dissolved in sterile saline containing $0.1 \%$ endotoxin-free bovine serum albumin (BSA, A-8806, Sigma), and the same solution of BSA (solvent) was used as a vehicle. IL-10 $(0.03 \mu \mathrm{g}$ in $5 \mu \mathrm{L}$ of sterile saline containing $0.1 \% \mathrm{BSA}$ ) or vehicle was intrathecally injected daily (intrathecal administration by lumbar puncture) 1 hour before the mechanical hypersensitivity test to measure the anti-allodynic effect of IL-10 in the CRPS mouse model for 7 days (Figure 1A). The dose for IL-10 was determined based on the previous studies. ${ }^{11,14}$

\section{Behavioral testing Mechanical hypersensitivity}

The $50 \%$ threshold to withdraw was measured by application of calibrated monofilaments (von Frey hairs; Stoelting, Wood Dale, IL, USA) to the plantar surface of the hind paw. ${ }^{15}$ The intensity of stimulus ranged from 0.004 to $1.7 \mathrm{~g}$, corresponding to the number of filaments $(1.65,2.36,2.44,2.83,3.22$, $3.61,3.84,4.08,4.17$, and 4.31).

\section{Rotarod}

An accelerating rotarod (Harvard Apparatus, Holliston, MA, USA) was used for measurement of the locomotor capacity of the mice. The task involves a speed ramp from 0 to 30 rotations per minute for 60 seconds, followed by an additional 240 seconds at the maximal speed. The latency to fall was measured for the first exposure to the rotarod, in addition to five consecutive trials (30 minutes inter-trial interval).

\section{Hind paw volume}

The thickness, lengths of the long axis (longitudinal; excluding dactyl region), and short axis of the bilateral hind paws were measured using an electronic digital caliper (Mitutoyo, Tokyo, Japan) and recorded at 3, 4, and 7 weeks. The paw 
A

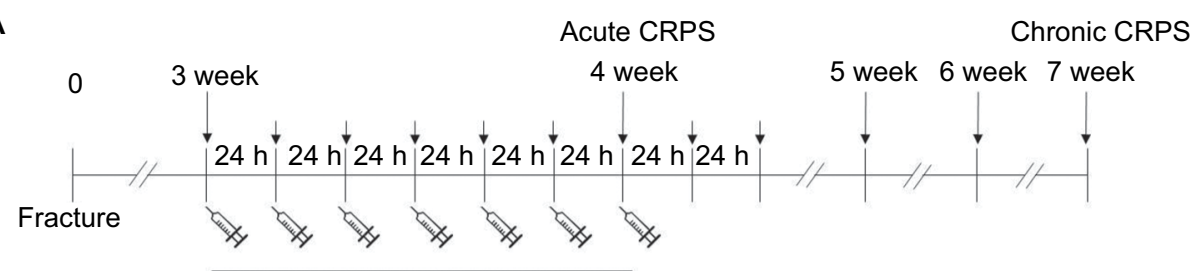

BSA or IL-10 daily intrathecal injection
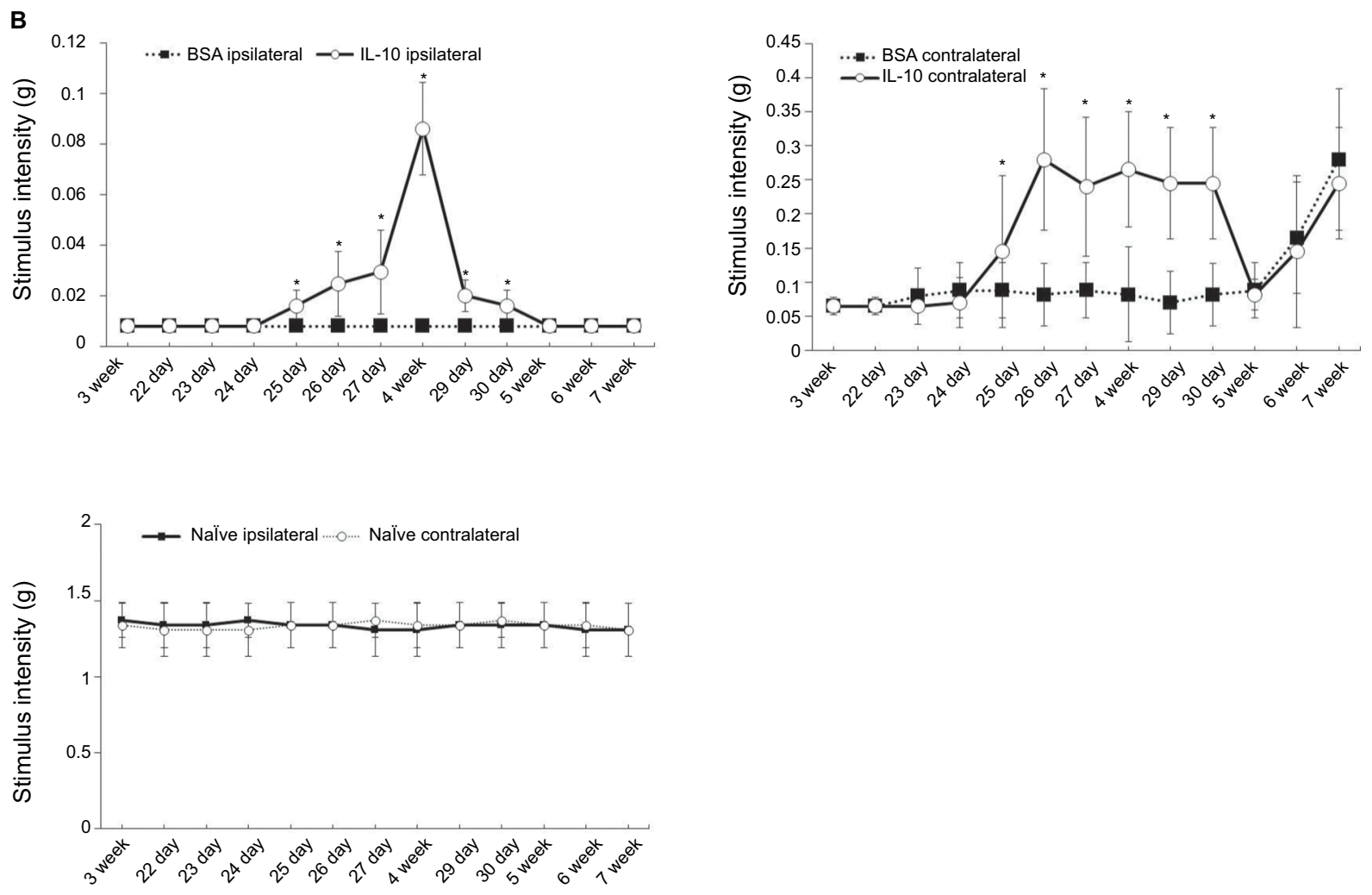

Figure I Time course of ipsilateral (fractured) and contralateral paw withdrawal thresholds up to 7 weeks after fracture (A). Recombinant mouse IL-10 or BSA was administered daily for 7 days through intrathecal injection. The intrathecal administration of recombinant IL-10 for 7 days resulted in increased mechanical thresholds of ipsilateral hind limbs when administered 3 weeks after fracture. However, early intervention of IL-10 to reduce central sensitization at the acute stage of CRPS could not make a significant improvement in mechanical allodynia of the ipsilateral paw at 7 weeks after fracture (B). $* P<0.05$ vs BSA-injected group.

Abbreviations: BSA, bovine serum albumin; CRPS, complex regional pain syndrome; IL-10, interleukin-10.

volume was calculated as described in a previous report ${ }^{16}$ : paw volume $\left(\mathrm{mm}^{3}\right)=1 / 2$ (length of short axis $\times$ length of long axis $\times$ thickness of paw).

\section{Immunohistochemistry}

Under anesthesia, mice were perfused through the aorta with $0.1 \mathrm{M}$ PBS followed by $4 \%$ paraformaldehyde fixative. The lumbar spinal cord was dissected out and post-fixed overnight. Then, the specimens were cryoprotected in $30 \%$ sucrose in $0.1 \mathrm{M}$ PBS at $4^{\circ} \mathrm{C}$. To prepare $30 \mu \mathrm{m}$ sections of lumbar spinal cord, a cryostat was used and the section of spinal cord was placed in PBS and stained with immunoperoxidase, as described previously. ${ }^{17}$ At room temperature, cryostat sections were blocked with $4 \%$ normal serum in $0.3 \%$ Triton X-100 for 1 hour. Then, cryostat sections were hybridized with primary antibodies (rabbit anti-mouse glial fibrillary acidic protein [GFAP] antibody [1:1000]; Dako, Carpinteria, CA, USA) and rabbit anti-mouse Iba-1 antibody ([1:1000]; Wako, Osaka, Japan) overnight at $4^{\circ} \mathrm{C}$. Then, cryostat sections were washed with PBS containing $0.1 \%$ Tween 20 (PBS-T) and incubated at room temperature with biotinylated secondary antibodies (Vector Laboratories, Burlingame, CA, USA) at 1:200 dilution. The cryostat sections were incubated in an avidin-biotin-peroxidase complex (1:100 dilution; ABC Elite; Vector Laboratories) for 
1 hour at room temperature after being washed several times in PBS-T. The horseradish peroxidase reaction was developed with $0.1 \mathrm{M}$ Tris-buffered saline ( $\mathrm{pH} 7.4$ ) containing $0.05 \%$ 3,3'-Diaminobenzidine tetrahydrochloride. Then, the cryostat sections were dehydrated, mounted on glass microscope slides, and cover-slipped. Iba1+ and GFAP+ cells were quantified in Image J (National Institute of Health, Bethesda, MD, USA).

\section{Real-time polymerase chain reaction (RT-PCR)}

Mice were anesthetized and perfused with PBS to remove blood. Then, the lumbar spinal cord was rapidly dissected out. The spinal cord at L4-L5 was divided into two constituent parts; ipsilateral and contralateral spinal cord. Then, these were frozen in liquid nitrogen and homogenized in TRIzol reagent (Life Technologies, Carlsbad, CA, USA). Total RNA $(2 \mu \mathrm{g})$ from each sample was reverse-transcribed into cDNA using a First Strand cDNA synthesis kit (MBI Fermentas, Hanover, Germany). Using the one-step SYBR ${ }^{\circledR}$ PrimeScript $^{\mathrm{TM}}$ RT-PCR kit (Perfect Real-Time; Takara Bio Inc., Tokyo, Japan) and the ABI Prism ${ }^{\circledR} 7000$ sequence detection system (Applied Biosystems, Foster City, CA, USA), real-time PCR was performed according to the manufacturer's instructions. The $2^{-\Delta \Delta C T}$ method was used to calculate relative changes in gene expression, ${ }^{18}$ and glyceraldehyde3-phosphate dehydrogenase was used as a control. The primer nucleotide sequences are listed in Table S1.

\section{Statistical analysis}

Statistical analyses were performed using SPSS for Windows and R package for Windows (version 2.15.2, R Foundation for Statistical Computing, Vienna, Austria). All results are presented as means \pm standard error of the mean (SEM). A two-tailed Student's $t$-test was employed for comparisons of two groups. Differences with $p$-values of $<0.05$ were considered statistically significant.

\section{Results}

Intrathecal recombinant IL-I0 (rIL- I0) administration was anti-allodynic in the acute stage ( 3 weeks) of the CRPS mouse model

The intrathecal administration of rIL-10 for 1 week resulted in increased mechanical thresholds of bilateral hind limbs when administered 3 weeks after fracture (Figure 1B). Increases in mechanical thresholds began at 4 days after intrathecal administration of rIL-10, with the best improvement at 1 week after administration, and the effect was maintained for 3 days after the injection was completed (Figure 1B).

\section{Intrathecal rIL-10 administration has no effect on decreasing the volume of the hind paw in the acute stage of the CRPS mouse model}

The volume of the ipsilateral hind paw at 3 weeks after fracture was significantly larger than that at 4 weeks after fracture in both IL-10- and BSA-injected groups (Figure 2A: $\mathrm{a}, \mathrm{b}, \mathrm{d}, \mathrm{e} ; 2 \mathrm{~B})$. However, there was no significant difference of volume in the ipsilateral hind paw at 4 weeks after fracture between IL-10- and BSA-injected groups (Figure 2A: $\mathrm{d}, \mathrm{e})$. The volume of the contralateral hind paw after fracture was not significantly different between the IL-10- and the BSA-injected groups at 4 weeks (Figure 2A: c, f; 2B). In addition, there was no significant difference of hind paw volume between naïve and 4 weeks after fracture (Figure 2C). This may mean that the intrathecal rIL-10 administration did not have a significant effect on the inflammation of the peripheral tissue.

\section{Anti-allodynic effects of intrathecal rIL- 10 administration developed by modulating microglial activation and decreasing tachykinin receptor I (TACRI) expression in the spinal cord}

In the immunohistochemistry at 4 weeks after fracture, there was a significant decrease in Iba $1+$ cells in the dorsal horn of the spinal cord of the IL-10-injected group compared with the BSA-injected group (Figure 3A: a-1; 3B). However, there was a significant decrease in GFAP+ cells in the dorsal horn of the spinal cord of the IL-10-injected group compared with the BSA-injected group (Figure S1). In real-time PCR at 4 weeks after fracture, the IL-10-injected group showed a significant decrease in the RNA level of mIL-1 $\beta$, mouse tumor necrosis factor-alpha (mTNF- $\alpha$ ), and mTACR1 of the ipsilateral spinal cord, although mIL- $1 \beta$, mTNF- $\alpha$, mGFAP, and mTACR1 were significantly increased in both groups compared with the ipsilateral spinal cord of the naive mouse (Figures 4 and 5).

\section{Intrathecal rIL- 10 administration in acute stage of CRPS mouse model cannot prevent the transition to the chronic stage of CRPS}

The anti-allodynic effect did not differ between the BSA and IL-10 treated groups after 1 week of stopping the injection, and mechanical hypersensitivity persisted until the seventh week in both groups (Figure 1B). In 

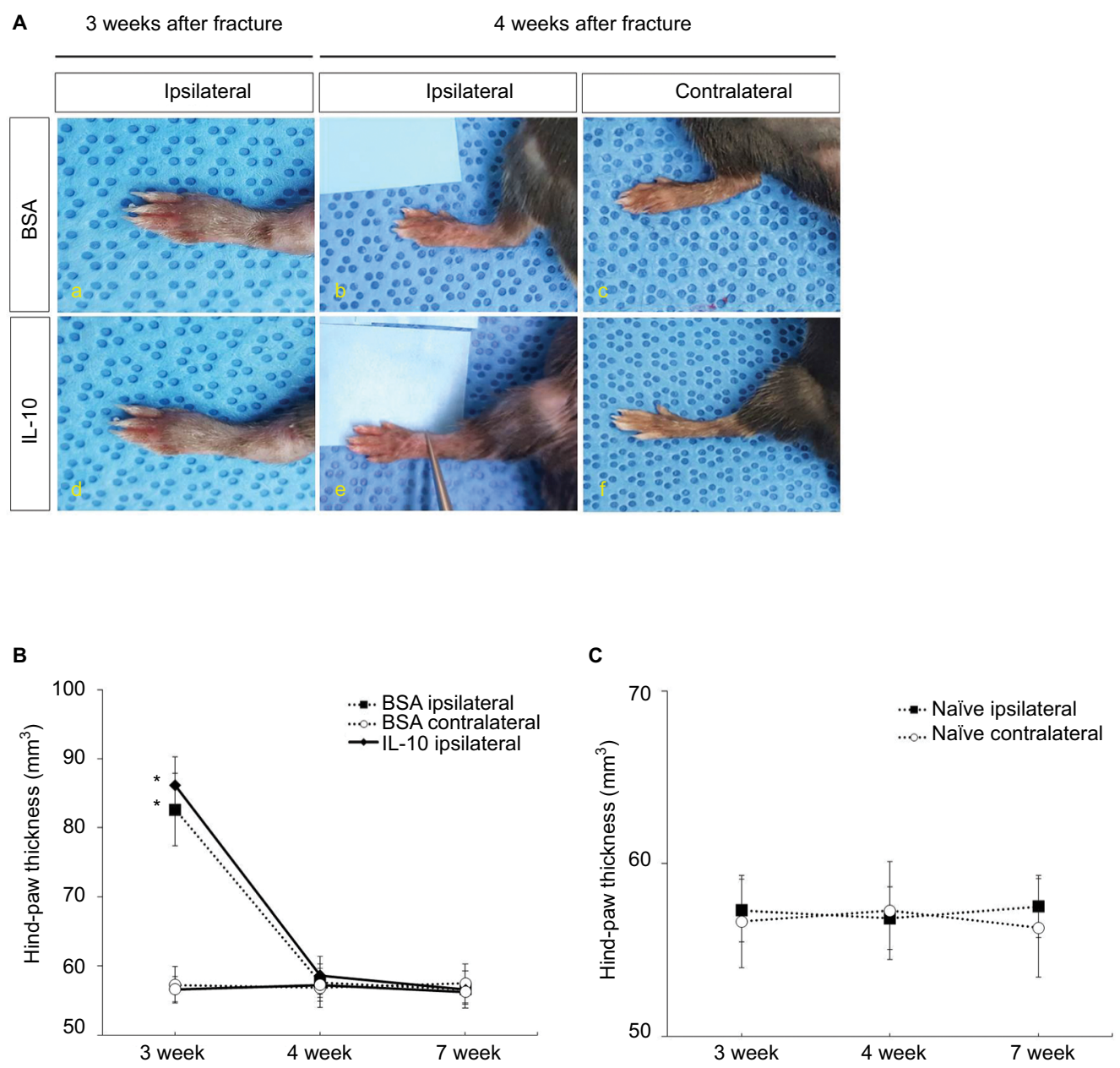

Figure 2 The image of bilateral hind paw in CRPS mice at 4 weeks (A); (a-c) BSA, (d-f) IL-I0. The volume of bilateral hind paw in CRPS mice at 3, 4, and 7 weeks (B). The volume of bilateral hind paw in naïve mice at 3,4 , and 7 weeks (C).

Abbreviations: BSA, bovine serum albumin; CRPS, complex regional pain syndrome; IL-I0, interleukin- 10.

immunohistochemistry, Iba1+ cells were not significantly different between the two groups at the dorsal horn of the bilateral spinal cord at 7 weeks after fracture (Figure 3A: $\mathrm{m}-\mathrm{t}$; 3B) In addition, there were also no significant differences in the RNA level of IL-1 $\beta$, TNF- $\alpha$ and TACR1 in real-time PCR, although mIL- $1 \beta$, mTNF- $\alpha$, mGFAP, and mTACR 1 were significantly increased in both groups compared with the ipsilateral spinal cord of the naïve mouse (Figures 4, 5) Considering this, the intrathecal administration of rIL-10 for 1 week had anti-allodynic effects, but did not prevent the transition to the chronic stage of CRPS from the acute stage.

At the 7-week time point, we observed no significant changes among naïve, BSA-treated, and IL-10 treated mice in the motor performance test. These data suggest that functional deficits caused by fracture are not easily observed at these time points (although there may be a tendency to reduce motor performance) (Figure S2).

\section{Discussion}

In previous studies of neuropathic pain or the CRPS rodent model, it has been reported that activated glial cells in the spinal cord can release pro-inflammatory cytokines, which initiate a signal transduction cascade and cause the release of excitatory amino acids and promote pain transmission. ${ }^{2,19}$ IL-10 is a potent anti-inflammatory cytokine, and it inhibits the synthesis of pro-inflammatory cytokines, such as TNF- $\alpha$ and IL- $1 \beta$ by activated microglia. ${ }^{20}$ By inhibiting the release of the pro-inflammatory cytokines TNF- $\alpha$ and IL-1 $\beta$ by activated microglia, it has already been reported that IL-10 possesses anti-allodynic or anti-hyperalgesic effects. ${ }^{11,12,14}$ The anti-allodynic effect of IL-10 is consistent 


\section{A}
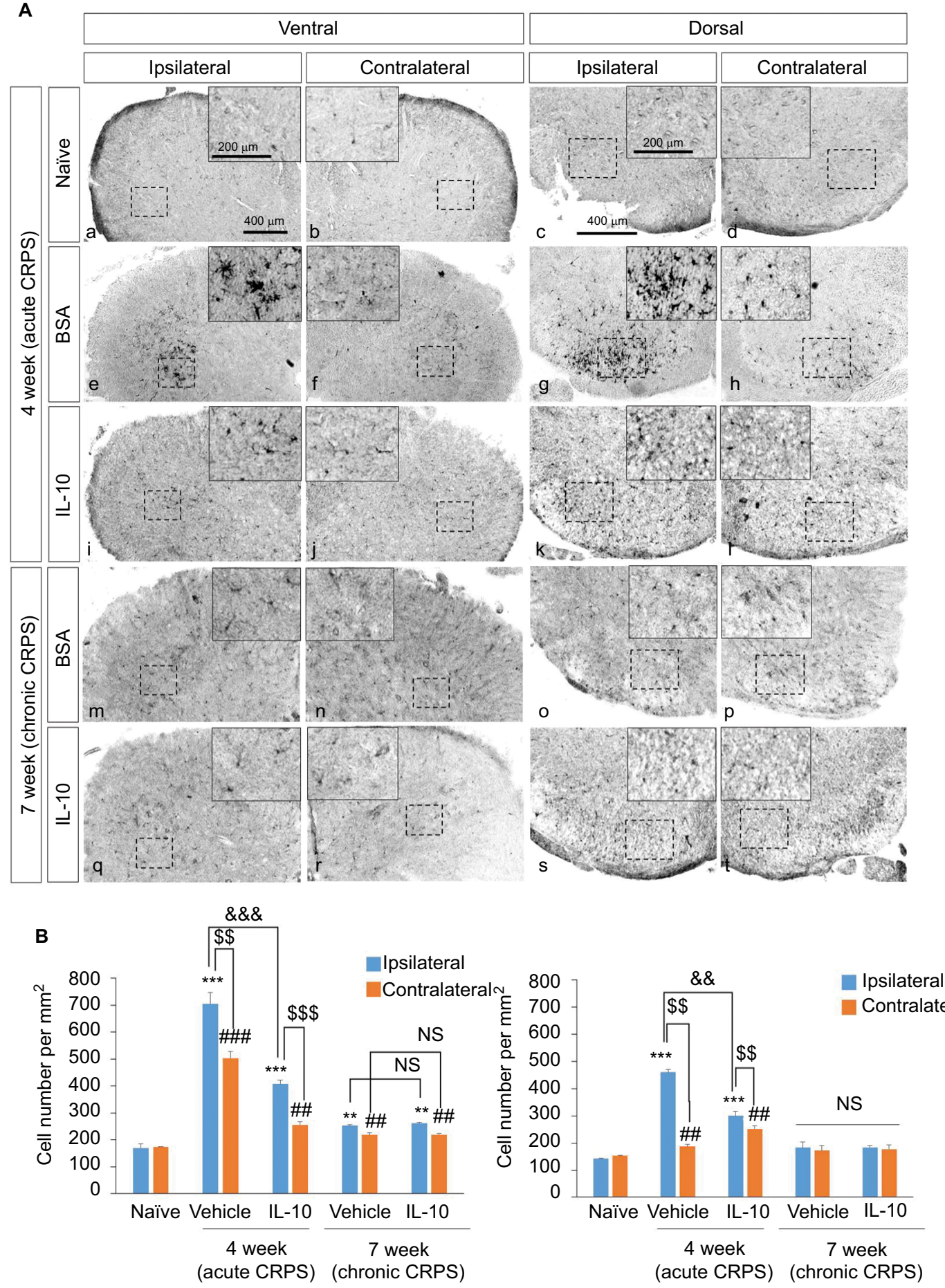

Figure 3 Effect of IL-10 on the activation of microglia in CRPS mouse model at 4 and 7 weeks after fracture.

Notes: (A) At 4 weeks after fracture, lba-l was detected in the spinal dorsal (a, b) and ventral horn (c, d) of naive mice, in the spinal dorsal (e, f) and ventral horn ( $\mathrm{g}$, h) of BSA-injected group, and in the spinal dorsal $(\mathrm{i}, \mathrm{j})$ and ventral horn $(\mathrm{k}, \mathrm{I})$ of the IL-I0-injected group. In addition, Iba- I was also detected in the spinal dorsal ( $\mathrm{m}, \mathrm{n}$ ) and ventral horn (o, p) of BSA-injected group, and in the spinal dorsal (q, r) and ventral horn (s, t) of the IL-I0-injected group. Scale bar, $400 \mu \mathrm{m}$. (B) Quantified immunohistochemical data for Iba-I are shown in the bar graphs, which display the number of lba-I-positive cells in the dorsal and ventral horn of the spinal cord at 4 weeks after fracture. Results are presented as mean \pm standard error of the mean. ${ }^{* * * P}<0.001$ vs naïve (ipsilateral), ${ }^{* *} P<0.05$ vs naïve (ipsilateral), ${ }^{\# \#} P<0.001$ vs naïve (contralateral), ${ }^{\#} P<0.05$ vs naïve (contralateral), ${ }^{\$ \$} \$<0.00$ I vs ipsilateral, ${ }^{\$ \$} P<0.05$ vs ipsilateral, ${ }^{8} \& \& P<0.001$ vs vehicle, ${ }^{2 \&} P<0.05$ vs vehicle.

Abbreviations: BSA, bovine serum albumin; CRPS, complex regional pain syndrome; IL-10, interleukin- I0; NS, not significant. 
4 week postinjection (CRPS)
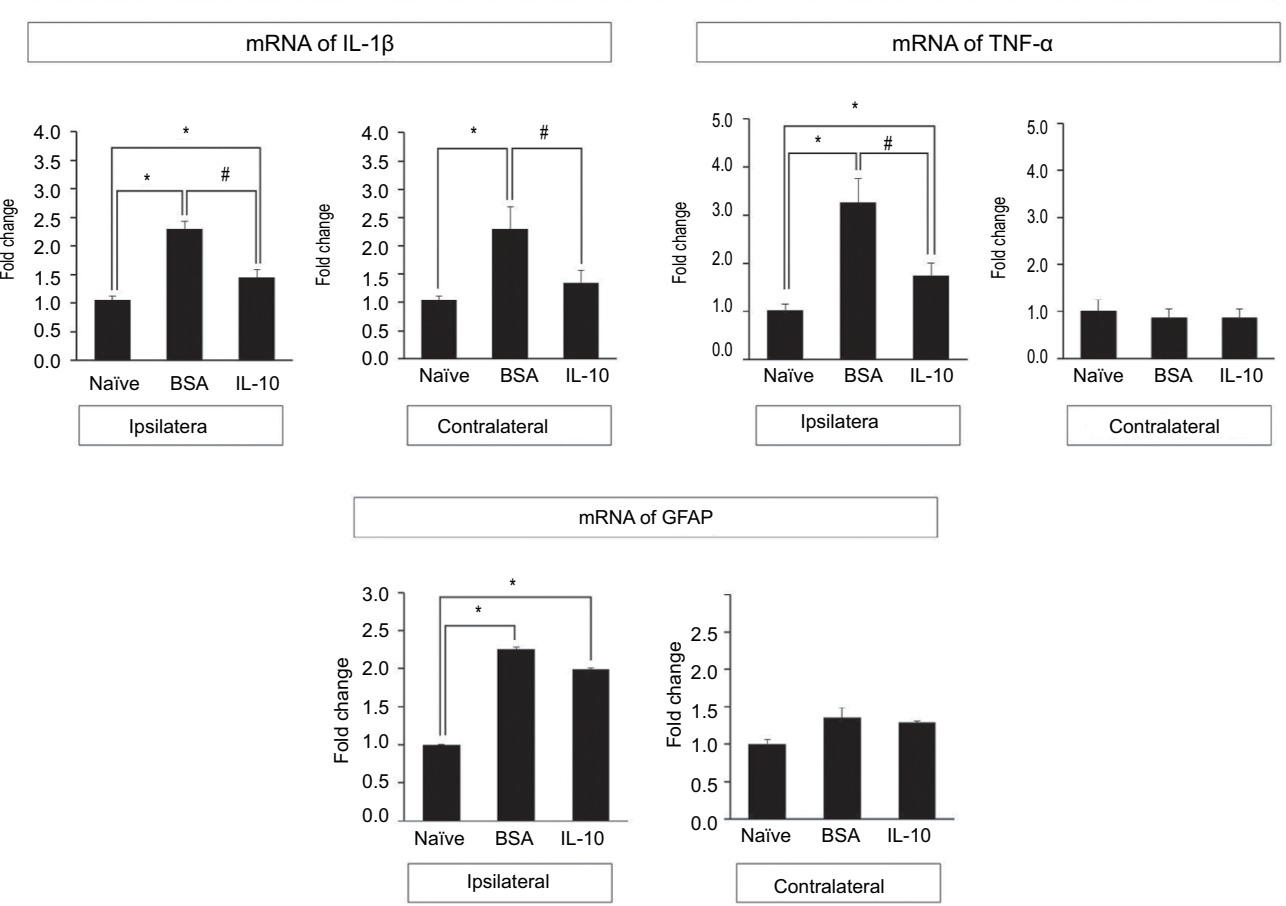

7 week p.i. (chronic CRPS)
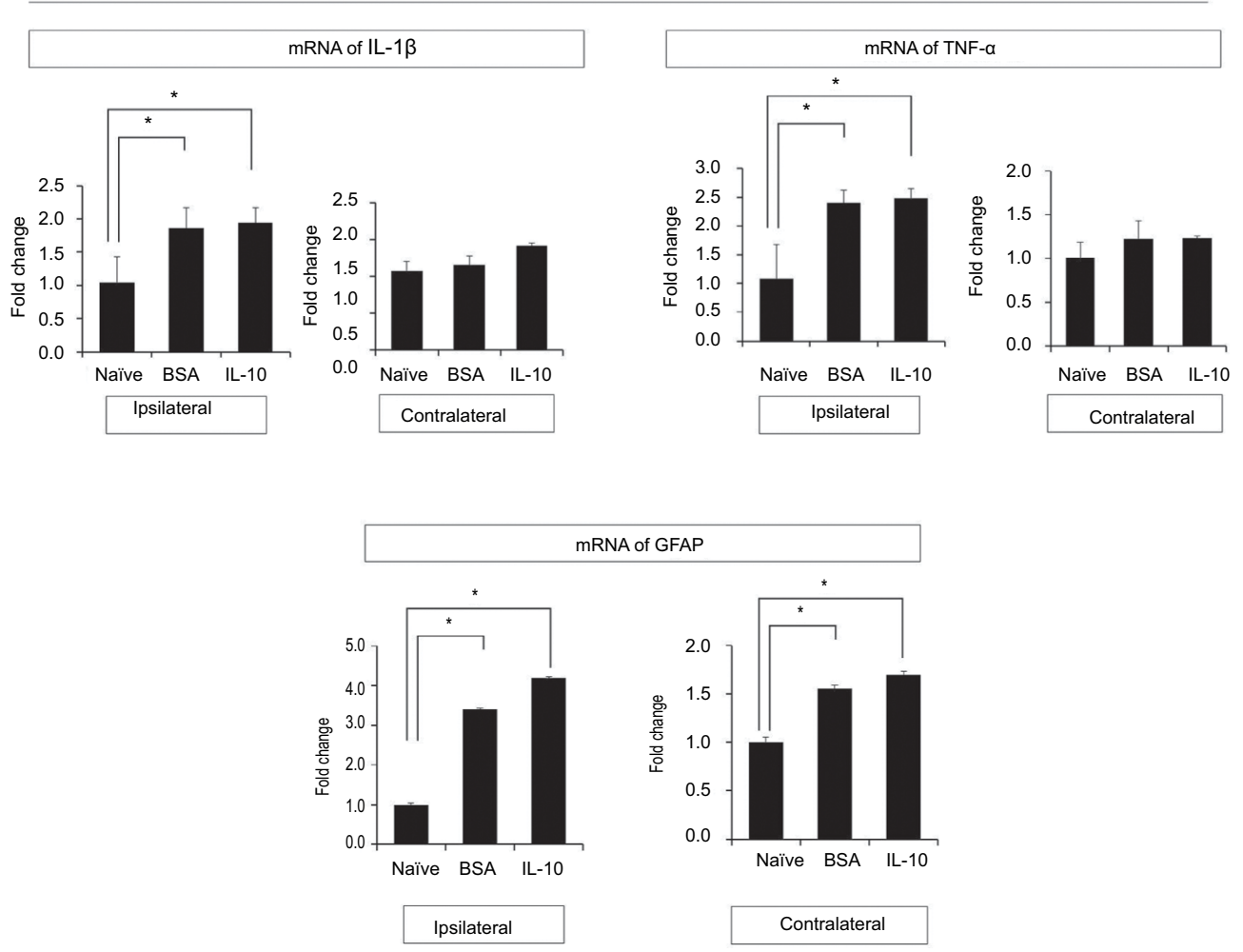

Figure 4 Real-time polymerase chain reaction results of spinal cord at 4 and 7 weeks after fracture.

Notes: Compared with the naïve group, mRNA expression of TNF- $\alpha$, IL-I $\beta$, GFAP were increased in both the IL-I0- and BSA-injected groups. Intrathecally injected IL-I0 reduced the mRNA expression of TNF- $\alpha$ and IL-I $\beta$ in the ipsilateral spinal cord of the CRPS mouse compared to the BSA group. In the contralateral spinal cord, the mRNA expressions of TNF- $\alpha$ and IL-I $\beta$ also decreased more in the IL-I0-injected group than in the BSA group. However, intrathecally injected IL-I0 could not reduce the mRNA expression of GFAP of the ipsilateral spinal cord compared with the BSA-injected group. All data points are expressed as the mean \pm standard error of the mean. $* P<0.05$ vs naïve group, ${ }^{\# P}<0.05$ vs BSA-injected group.

Abbreviations: BSA, bovine serum albumin; CRPS, complex regional pain syndrome; GFAP, glial fibrillary acidic protein; IL-I0, interleukin-I0; TNF- $\alpha$, tumor necrosis factor- $\alpha$. 

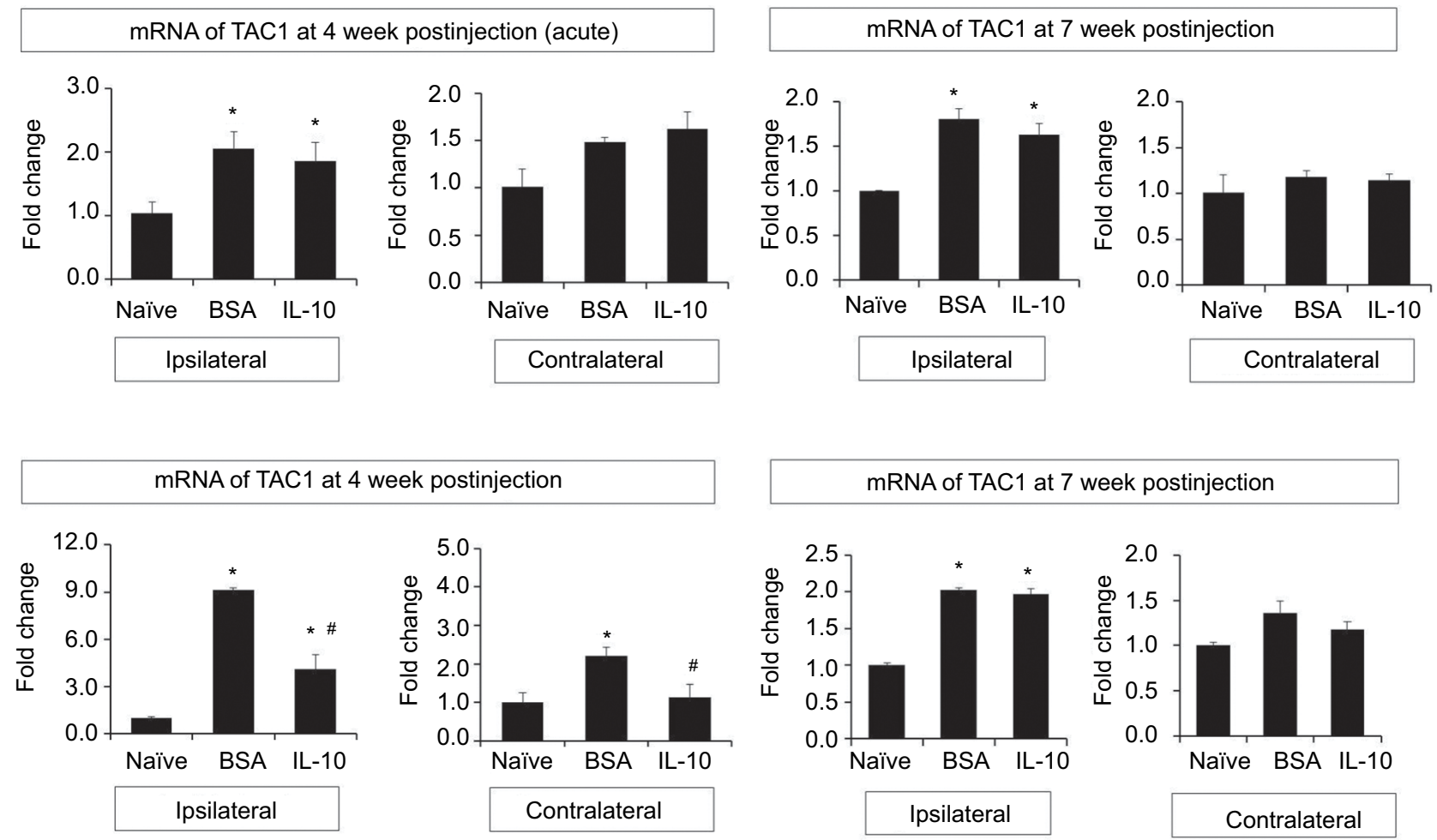

Figure 5 Real-time polymerase chain reaction results of spinal cord at 4 and 7 weeks after fracture.

Notes: Compared with the naive group, mRNA expression of TACI and TACRI were increased in both the IL-I0-injected and BSA groups. Intrathecally injected IL10 reduced the mRNA expression of TACRI in the ipsilateral spinal cord of the CRPS mouse compared to the BSA group. In the contralateral spinal cord, the mRNA expressions of TACR I also decreased more in the IL-I0-injected group than in the BSA group. However, intrathecally injected IL-I0 could not reduce the mRNA expression of TACI of ipsilateral spinal cord compared with the BSA-injected group. All data points are expressed as the mean \pm standard error of the mean. $* P<0.05$ vs naive group, ${ }^{\#} P<0.05$ vs $B S A$-injected group.

Abbreviations: BSA, bovine serum albumin; CRPS, complex regional pain syndrome; IL-I0, interleukin-I0; TACI, tachykinin I; TACRI, tachykinin receptor I.

with the results of our study, which showed a decrease in microglial activation (decrease of microglial marker, Iba 1) in immunohistochemistry and a significant decrease in mRNA expression of TNF- $\alpha$ and IL- $1 \beta$ in the spinal cord of the IL-10-injected group. In addition, the results of this study also showed that mRNA expression of TACR1 (neurokinin 1 receptor [NK1R]) was significantly decreased in the spinal cord of the IL-10-injected group, unlike substance P, which did not show a significant difference from the BSA-injected group in the IL-10-injected group.

Substance $\mathrm{P}$ is an 11-amino acid neuropeptide present in the peripheral and central terminals of non-myelinated primary sensory neurons. ${ }^{21,22}$ The actions of substance $\mathrm{P}$ are mainly mediated by NK1R, encoded by the tachykinin 1 receptor (tac1r) gene, ${ }^{21,22}$ and glia cells, such as microglia and astrocytes as well as neuron express substance P NK1 receptors. ${ }^{23-26}$

It is known that substance P stimulates glia to express TNF-a, IL-1, and IL-6 pro-inflammatory cytokines. ${ }^{27-30} \mathrm{In}$ previous studies of the fracture/casting CRPS rodent model, substance P mRNA and protein expression in the ipsilateral L4-L5 dorsal root ganglia and sciatic nerve, and expression of NK1 receptor in the lumbar spinal cord were increased, indicating amplification of substance $\mathrm{P}$ spinal signaling in CRPS.$^{31}$ Moreover, many previous studies using transgenic substance P-deficient or NK1 receptor-deficient animal models have demonstrated that an increase of a pro-inflammatory cytokine, such as TNF- $\alpha$ and IL- $1 \beta$, in the spinal glia cells is dependent on substance signaling. ${ }^{2,21,31}$ Moreover, a proinflammatory cytokine increase for substance $P$ exacerbates neuro-inflammation by increasing the expression of NK1R in glia cells (Figure 6). However, the administration of IL-10 is thought to have an anti-allodynic effect, not only in inhibiting pro-inflammatory cytokines in activated microglia, but also in inhibiting the expression of NK1R in the CRPS mouse model, which is consistent with the results of our study and previous studies..$^{32,33}$

The present results demonstrate for the first time that intrathecal administration of rIL-10 can attenuate the mechanical allodynia in the CRPS mouse model. These 


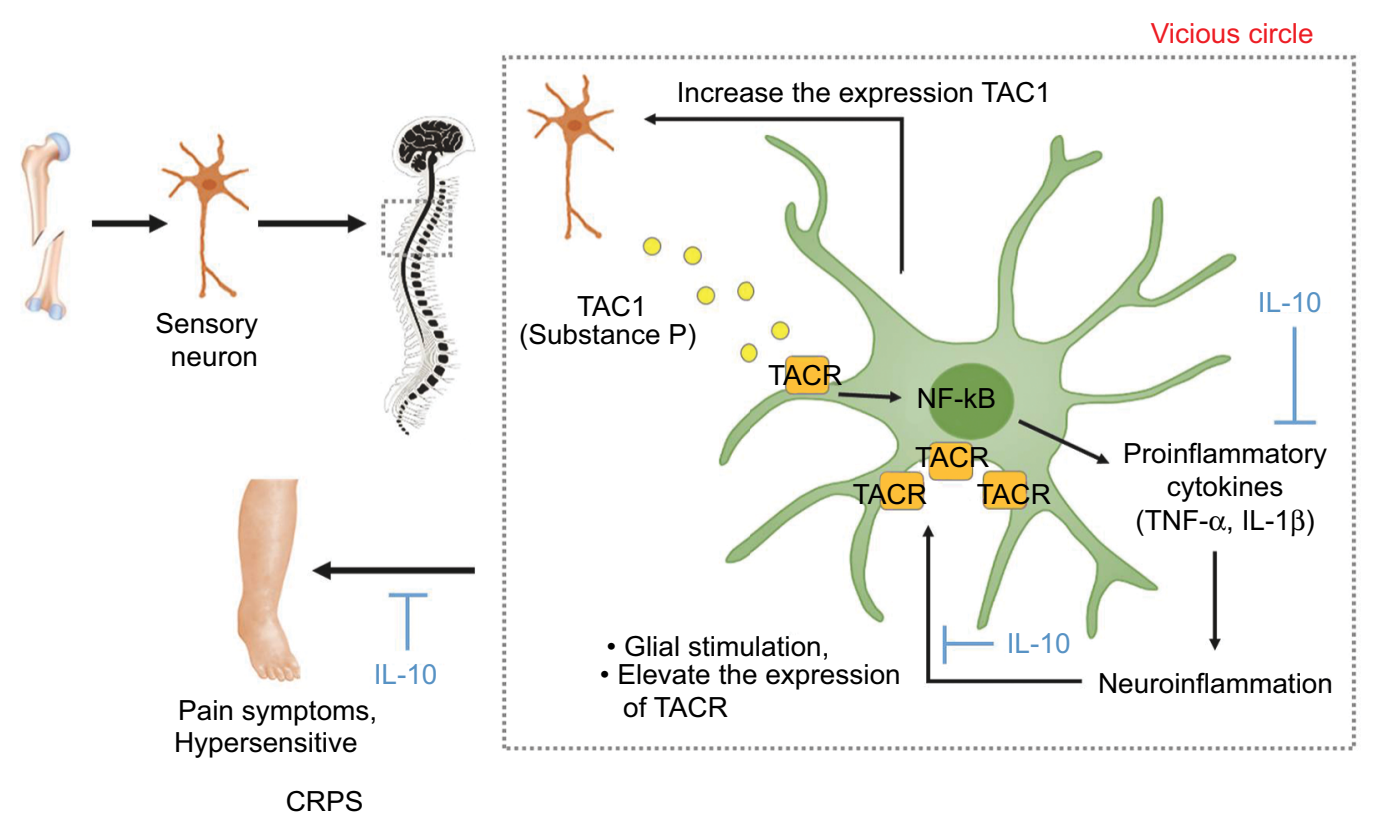

Figure 6 Substance P-mediated exacerbation of neuro-inflammation causes central sensitization in the CRPS mouse model.

Notes: The actions of substance $P$ are mainly mediated by NKIR, encoded by TACIR gene. Substance P-mediated by nociceptive sensory neurons due to peripheral nociceptive stimuli stimulated glia expression of pro-inflammatory cytokines, such as TNF- $\alpha$, IL-I $\beta$, and IL-6. These pro-inflammatory cytokines, which are increased by substance $\mathrm{P}$, also exacerbate neuro-inflammation by increasing the expression of NKI receptor in glia cells, and can elevate local production of substance P. This positive feedback loop would be anticipated to sensitize glia to this neuropeptide, thereby exacerbating central sensitization. Administration of IL-I0 is thought to have an antiallodynic effect, not only in inhibiting pro-inflammatory cytokines in activated microglia but also in inhibiting the expression of NKI receptors in the CRPS mouse model. Abbreviations: BSA, bovine serum albumin; CRPS, complex regional pain syndrome; IL-I0, interleukin-I0; NKIR, neurokinin I receptor; TACI, tachykinin I; TACRI, tachykinin receptor I; TNF- $\alpha$, tumor necrosis factor- $\alpha$.

results are similar to the anti-allodynic effect of IL-10 seen in previous neuropathic pain mouse models. ${ }^{11,12,14}$ However, unlike previous studies that focused on the reduction of proinflammatory cytokine secretion by IL-10, we have found that inhibition of substance $\mathrm{P}$ receptors (NK1 receptors) expression in microglia can be another mechanism of antiallodynic effect of intrathecal IL-10.

In our study, we also investigated whether early inhibition of neuro-inflammation by IL-10 administration, which is considered to be one of the important mechanisms in the generation of central sensitization, could prevent the transition to the chronic stage in the acute stage of CRPS. Although intrathecal administration of rIL-10 in the acute stage of CRPS led to a significant decrease in neuroinflammation through a decrease of significant microglial activation and pro-inflammatory cytokine and NK1R expression, it was not sufficient to prevent the transition to CRPS. This was probably due to two reasons: 1) an insufficient amount or duration of intrathecal IL-10 administration to prevent the transition to the chronic stage despite the effective decrease of neuro-inflammation in the acute stage of CRPS; 2) the possibility of central sensitization by mechanisms other than neuro-inflammation by spinal glial activation, such as supra-spinal structures. Considering that some clinical studies have shown evidence that other than the spinal cord, the prefrontal cortex, ${ }^{34}$ thalamus, ${ }^{8}$ and the basal ganglia, ${ }^{35}$ play an important role in the development of central sensitization of CRPS, further studies will be needed to elucidate the underlying mechanism of central sensitization other than neuro-inflammation in the CRPS.

Our study has some limitations. First, only a single dose of rIL-10 was administrated in this study. However, the dose of IL-10 in our study was determined on the basis of the dose of IL-10 with anti-allodynic effects in previous studies. In addition, this dose of IL-10 has also anti-allodynic effects in CRPS mice. Although administration of a higher dose of IL-10 may have increased the degree and duration of mechanical allodynia, it does not seem to prevent transition to the chronic stage of CRPS completely considering sustained increased substance $P$ level of the spinal cord in CRPS mice. Because the anti-inflammatory effect of IL-10, which 
can attenuate the neuro-inflammation of the spinal cord, is temporary, the continued secretion of substance $\mathrm{P}$ or other neurotransmitters in sensory afferent nerves at the spinal cord dorsal horn will exacerbate the neuro-inflammation again. However, considering the anti-allodynic effects of IL-10 in the CRPS mouse model, it may be more effective in reducing the allodynia of CRPS using gene therapy with adeno-associated virus. Second, we could not measure thermal allodynia of the hind paw. ${ }^{36}$ Because we referred to previous study of CRPS mice, which checked only mechanical allodynia for measurement of allodynia, we thought that mechanical allodynia and incapitance tests were sufficient to check the anti-nociceptive effects of IL-10. Considering previous studies in which IL-10 was effective for thermal allodynia in neuropathic pain rodent models, IL-10 appears to be effective in thermal allodynia in CRPS mice. ${ }^{37}$ In the future, additional studies, including thermal allodynia may be necessary to confirm the anti-thermal allodynic effect of IL-10 in CRPS mice.

In conclusion, our present results demonstrate that intrathecally administered rIL-10 attenuates mechanical allodynia in the CRPS mouse model. The effects of IL-10 may be due, at least in part, to the modulation of microglia activation, and the decrease in pro-inflammatory cytokines and NK1 receptors in the CRPS mouse spinal cords. However, these effects of IL-10 on allodynia in the acute stage of CRPS were not sufficient to prevent the transition to the chronic stage of CRPS. In the future, further studies on the mechanisms of central sensitization in CRPS will be necessary.

\section{Acknowledgments}

Special thanks to Jin Han for helping us write the manuscript. This research was supported by Basic Science Research Program through the National Research Foundation of Korea (NRF) funded by the Ministry of Science, ICT and Future Planning (NRF-2015R1D1A1A01056654 and NRF-2017R1D1A1B03033127).

\section{Disclosure}

The authors report no conflicts of interest in this work.

\section{References}

1. de Mos M, de Bruijn AG, Huygen FJ, Dieleman JP, Stricker BH, Sturkenboom MC. The incidence of complex regional pain syndrome: a population-based study. Pain. 2007;129(1-2):12-20.

2. Tajerian M, Leu D, Yang P, Huang TT, Kingery WS, Clark JD. Differential Efficacy of Ketamine in the Acute versus Chronic Stages of Complex Regional Pain Syndrome in Mice. Anesthesiology. 2015;123(6): 1435-1447.
3. Bruehl S. An update on the pathophysiology of complex regional pain syndrome. Anesthesiology. 2010;113(3):1-725.

4. Parkitny L, Mcauley JH, di Pietro F, et al. Inflammation in complex regional pain syndrome: a systematic review and meta-analysis. Neurology. 2013;80(1):106-117.

5. Gallagher JJ, Tajerian M, Guo T, et al. Acute and chronic phases of complex regional pain syndrome in mice are accompanied by distinct transcriptional changes in the spinal cord. Mol Pain. 2013;9:40.

6. Linnman C, Becerra L, Borsook D. Inflaming the brain: CRPS a model disease to understand neuroimmune interactions in chronic pain. $J$ Neuroimmune Pharmacol. 2013;8(3):547-563.

7. Banati RB. Neuropathological imaging: in vivo detection of glial activation as a measure of disease and adaptive change in the brain. $\mathrm{Br}$ Med Bull. 2003;65:121-131.

8. Banati RB, Cagnin A, Brooks DJ, et al. Long-term trans-synaptic glial responses in the human thalamus after peripheral nerve injury. Neuroreport. 2001;12(16):3439-3442.

9. Rasley A, Tranguch SL, Rati DM, Marriott I. Murine glia express the immunosuppressive cytokine, interleukin-10, following exposure to Borrelia burgdorferi or Neisseria meningitidis. Glia. 2006;53(6):583-592.

10. Pearse DD, Marcillo AE, Oudega M, Lynch MP, Wood PM, Bunge MB. Transplantation of Schwann cells and olfactory ensheathing glia after spinal cord injury: does pretreatment with methylprednisolone and interleukin-10 enhance recovery? $J$ Neurotrauma. 2004;21(9):1223-1239.

11. Lee BS, Jun IG, Kim SH, Park JY. Intrathecal gabapentin increases interleukin-10 expression and inhibits pro-inflammatory cytokine in a rat model of neuropathic pain. J Korean Med Sci. 2013;28(2):308-314.

12. Zheng W, Huang W, Liu S, et al. Interleukin 10 mediated by herpes simplex virus vectors suppresses neuropathic pain induced by human immunodeficiency virus gp120 in rats. Anesth Analg. 2014;119(3):693-701.

13. Song XJ, Huang ZJ, Song WB, et al. Attenuation Effect of Spinal Manipulation on Neuropathic and Postoperative Pain Through Activating Endogenous Anti-Inflammatory Cytokine Interleukin 10 in Rat Spinal Cord. J Manipulative Physiol Ther. 2016;39(1):42-53.

14. Lin SL, Tsai RY, Tai YH, et al. Ultra-low dose naloxone upregulates interleukin-10 expression and suppresses neuroinflammation in morphine-tolerant rat spinal cords. Behav Brain Res. 2010;207(1):30-36.

15. Chaplan SR, Bach FW, Pogrel JW, Chung JM, Yaksh TL. Quantitative assessment of tactile allodynia in the rat paw. J Neurosci Methods. 1994;53(1):55-63.

16. Cho YH, Chung IK, Cheon WH, Lee HS, Ku SK. Effect of DHU001, a Polyherbal Formula on Formalin-induced Paw Chronic Inflammation of Mice. Toxicol Res. 2011;27(2):95-102.

17. Jeon SM, Sung JK, Cho HJ. Expression of monocyte chemoattractant protein-1 and its induction by tumor necrosis factor receptor 1 in sensory neurons in the ventral rhizotomy model of neuropathic pain. Neuroscience. 2011;190:354-366.

18. Livak KJ, Schmittgen TD. Analysis of relative gene expression data using real-time quantitative PCR and the 2(-Delta Delta C(T)) Method. Methods. 2001;25(4):402-408.

19. Kawasaki Y, Xu ZZ, Wang X, et al. Distinct roles of matrix metalloproteases in the early- and late-phase development of neuropathic pain. Nat Med. 2008;14(3):331-336.

20. Zhang JM, An J. Cytokines, inflammation, and pain. Int Anesthesiol Clin. 2007;45(2):27-37.

21. Matak I, Tékus V, Bölcskei K, Lacković Z, Helyes Z. Involvement of substance $\mathrm{P}$ in the antinociceptive effect of botulinum toxin type A: Evidence from knockout mice. Neuroscience. 2017;358:137-145.

22. Nakagawasai O, Tadano T, Tan-No K, et al. Antinociceptive effect following dietary-induced thiamine deficiency in mice: involvement of substance P and somatostatin. Life Sci. 2001;69(10):1155-1166.

23. Torrens Y, Beaujouan JC, Saffroy M, Daguet de Montety MC, Bergström L, Glowinski J. Substance P receptors in primary cultures of cortical astrocytes from the mouse. Proc Natl Acad Sci U S A. 1986;83(23):9216-9220. 
24. Lin RC. Reactive astrocytes express substance-P immunoreactivity in the adult forebrain after injury. Neuroreport. 1995;7(1):310-312.

25. Lai JP, Zhan GX, Campbell DE, Douglas SD, Ho WZ. Detection of substance $\mathrm{P}$ and its receptor in human fetal microglia. Neuroscience. 2000;101(4):1137-1144.

26. Rasley A, Bost KL, Olson JK, Miller SD, Marriott I. Expression of functional NK-1 receptors in murine microglia. Glia. 2002;37(3): 258-267.

27. Martin FC, Anton PA, Gornbein JA, Shanahan F, Merrill JE. Production of interleukin-1 by microglia in response to substance P: role for a non-classical NK-1 receptor. J Neuroimmunol. 1993;42(1):53-60.

28. Luber-Narod J, Kage R, Leeman SE. Substance P enhances the secretion of tumor necrosis factor-alpha from neuroglial cells stimulated with lipopolysaccharide. J Immunol. 1994;152(2):819-824.

29. Fiebich BL, Schleicher S, Butcher RD, Craig A, Lieb K. The neuropeptide substance $\mathrm{P}$ activates $\mathrm{p} 38$ mitogen-activated protein kinase resulting in IL-6 expression independently from NF-kappa B. J Immunol. 2000;165(10):5606-5611.

30. Zhu J, Qu C, Lu X, Zhang S. Activation of microglia by histamine and substance P. Cell Physiol Biochem. 2014;34(3):768-780.
31. Li WW, Guo TZ, Shi X, et al. Substance P spinal signaling induces glial activation and nociceptive sensitization after fracture. Neuroscience. 2015;310:73-90.

32. Weinstock JV, Blum A, Metwali A, Elliott D, Bunnett N, Arsenescu R. Substance P regulates Th1-type colitis in IL-10 knockout mice. $J$ Immunol. 2003;171(7):3762-3767.

33. Johnson MB, Young AD, Marriott I. The Therapeutic Potential of Targeting Substance P/NK-1R Interactions in Inflammatory CNS Disorders. Front Cell Neurosci. 2016;10:296.

34. Gieteling EW, van Rijn MA, de Jong BM, et al. Cerebral activation during motor imagery in complex regional pain syndrome type 1 with dystonia. Pain. 2008;134(3):302-309.

35. Park D. Pramipexole-induced limb dystonia and its associated complex regional pain syndrome in idiopathic Parkinson's disease: A case report Medicine. 2017;96(28):e7530.

36. Wei T, Guo TZ, Li WW, Kingery WS, Clark JD. Acute versus chronic phase mechanisms in a rat model of CRPS. J Neuroinflammation. 2016;13:14.

37. Milligan ED, Sloane EM, Langer SJ, et al. Controlling neuropathic pain by adeno-associated virus driven production of the anti-inflammatory cytokine, interleukin-10. Mol Pain. 2005;1:9. 


\section{Supplementary materials}

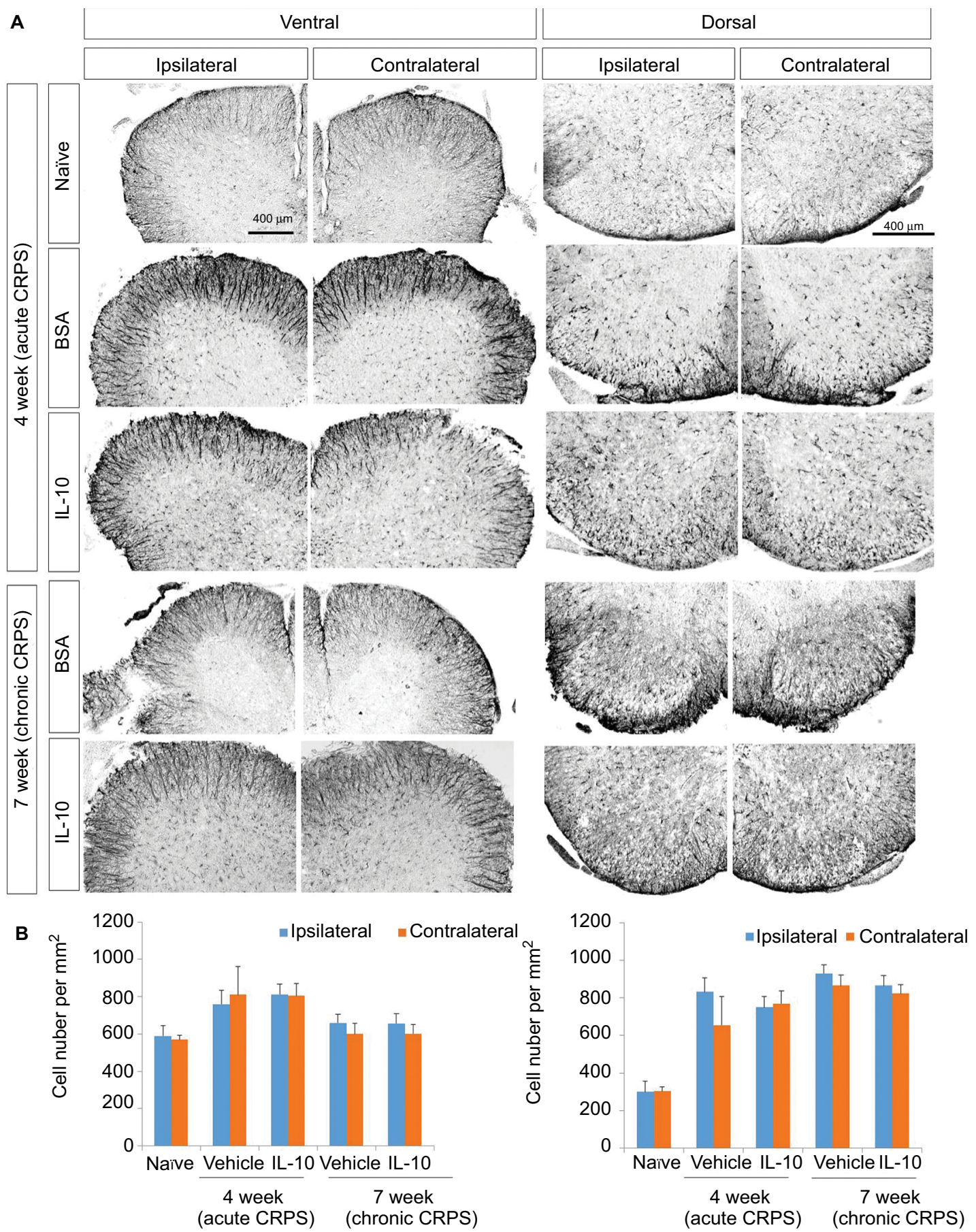

Figure SI Effect of IL-10 on the activation of astrocytes in CRPS mouse model at 4 and 7 weeks after fracture.

Notes: (A) GFAP was detected in the spinal dorsal and ventral horn of naive mice, in the spinal dorsal and ventral horn of BSA-injected group, and in the spinal dorsal and ventral horn of the IL-10-injected group. Scale bar, $400 \mu \mathrm{m}$. (B) Quantified immunohistochemical data for Iba-I are shown in the bar graphs, which display the number of GFAP-positive cells in the dorsal and ventral horn of the spinal cord at 4 weeks after fracture. Results are presented as mean \pm standard error of the mean.

Abbreviations: BSA, bovine serum albumin; CRPS, complex regional pain syndrome; GFAP, glial fibrillary acidic protein; IL-10, interleukin-10. 


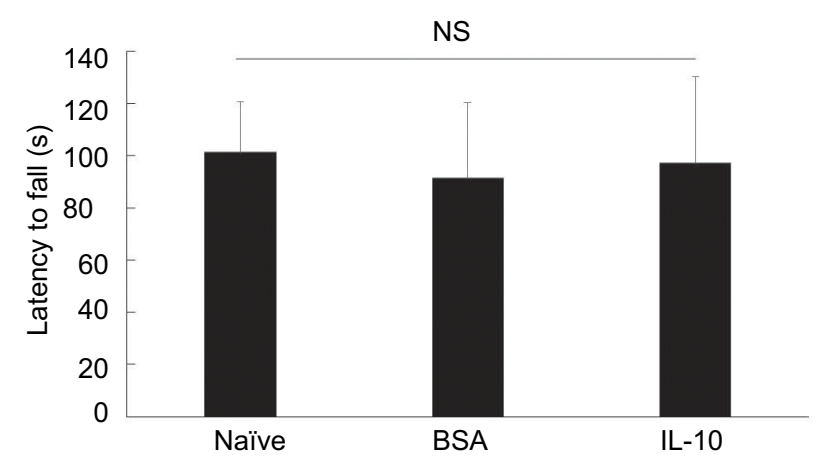

Figure S2 The results of Rotarod test in naïve and CRPS mice at 7 weeks.

Abbreviations: BSA, bovine serum albumin; CRPS, complex regional pain syndrome; IL-10, interleukin-10; NS, not significant.

Table SI Nucleotide sequences of the primers used in RT-PCR

\begin{tabular}{|c|c|c|}
\hline Mouse cDNA & RT-PCR methods & Primer sequences \\
\hline \multirow[t]{2}{*}{ GAPDH } & Real-time & F, 5'-TGG GCT ACA CTG AGC ACC AG-3' \\
\hline & & R, 5'-GGG TGT CGC TGT TGA AGT CA-3' \\
\hline \multirow[t]{2}{*}{ IL-I $\beta$} & Real-time & F, 5'-AAG TTG ACG GAC CCC AAA AGA T-3' \\
\hline & & R, 5'-TGT TGA TGT GCT GCT GCG A-3' \\
\hline \multirow[t]{2}{*}{ TNF- $\alpha$} & Real-time & F, 5'-ATG GCC TCC CTC TCA GTT C-3' \\
\hline & & R, 5'-TTG GTG GTT TGC TAC GAC GTG-3' \\
\hline \multirow[t]{2}{*}{ GFAP } & Real-time & F, 5'-GAA GAA AAC CGC ATC ACC AT-3' \\
\hline & & R, 5'-GCA CAC CTC ACA TCA CAT CC- $3^{\prime}$ \\
\hline \multirow[t]{2}{*}{$\mathrm{TACl}$} & Real-time & F, 5'-TTT GCA GAG GAA ATC GGT GCC AAC-3' \\
\hline & & R, 5'-GGC ATT GCC TCC TTG ATT TGG TCA-3' \\
\hline \multirow[t]{2}{*}{ TACRI } & Real-time & F, 5'-CTG GAA AGA GGA GCC TTG TG-3' \\
\hline & & R, 5'-CTG AGA CGG AAA GGA ACA-GC-3' \\
\hline
\end{tabular}

Abbreviations: GAPDH, glyceraldehyde 3-phosphate dehydrogenase; GFAP, glial fibrillary acidic protein; IL-I $\beta$, interleukin-I $\beta$; RT-PCR, real-time polymerase chain reaction; TACI, tachykinin I; TACRI, tachykinin receptor I; TNF- $\alpha$, tumor necrosis factor- $\alpha$.

Journal of Pain Research

\section{Publish your work in this journal}

The Journal of Pain Research is an international, peer reviewed, open access, online journal that welcomes laboratory and clinical findings in the fields of pain research and the prevention and management of pain. Original research, reviews, symposium reports, hypothesis formation and commentaries are all considered for publication.

\section{Dovepress}

The manuscript management system is completely online and includes a very quick and fair peer-review system, which is all easy to use. Visit http://www.dovepress.com/testimonials.php to read real quotes from published authors. 